\title{
Systematic histopathological analysis of different 5-aminolevulinic acid-induced fluorescence levels in newly diagnosed glioblastomas
}

\author{
Barbara Kiesel, MD, ${ }^{1,2,5}$ Mario Mischkulnig, MD, ${ }^{1}$ Adelheid Woehrer, MD, PhD, ${ }^{2,5}$ \\ Mauricio Martinez-Moreno, MD, ${ }^{1}$ Matthias Millesi, MD, ${ }^{1,5}$ Ammar Mallouhi, MD, ${ }^{3,5}$ \\ Thomas Czech, MD, ${ }^{1,5}$ Matthias Preusser, MD, ${ }^{4,5}$ Johannes A. Hainfellner, MD, ${ }^{2,5}$ \\ Stefan Wolfsberger, MD, ${ }^{1,5}$ Engelbert Knosp, MD, ${ }^{1,5}$ and Georg Widhalm, MD, PhD ${ }^{1,5}$ \\ ${ }^{1}$ Department of Neurosurgery, ${ }^{2}$ nstitute of Neurology, Departments of ${ }^{3}$ Radiology and ${ }^{4}$ Medicine I, and ${ }^{5}$ Comprehensive Cancer \\ Center-Central Nervous System Tumours Unit (CCC-CNS), Medical University Vienna, Austria
}

OBJECTIVE Glioblastoma (GBM) is characterized by distinct intratumoral histopathological heterogeneity with regard to variable tumor morphology, cell proliferation, and microvascularity. Maximum resection of a GBM results in an improved prognosis and thus represents the aim of surgery in the majority of cases. Fluorescence-guided surgery using 5 -aminolevulinic acid (5-ALA) is currently widely applied for improved intraoperative tumor visualization in patients with a GBM. Three intratumoral fluorescence levels (i.e., strong, vague, or no fluorescence) can usually be distinguished during surgery. So far, however, their exact histopathological correlates and their surgical relevance have not been clarified sufficiently. Thus, the aim of this study was to systematically analyze tissue samples from newly diagnosed GBMs with different fluorescence levels according to relevant histopathological parameters.

METHODS This prospective study recruited patients who underwent 5-ALA fluorescence-guided resection of a newly diagnosed radiologically suspected GBM. Each patient received 5-ALA approximately 3 hours before surgery, and a modified neurosurgical microscope was applied for intraoperative visualization of 5-ALA-induced fluorescence. During surgery, tissue samples with strong, vague, or no fluorescence were collected. For each sample, the presence of tumor tissue, quality of tissue (compact, infiltrative, or no tumor), histopathological criteria of malignancy (cell density, nuclear pleomorphism, mitotic activity, and presence of microvascular proliferation/necrosis), proliferation rate (MIB-1 labeling index [LI]), and microvessel density (using CD34 staining) were investigated.

RESULTS Altogether, 77 patients with a newly diagnosed, histopathologically confirmed GBM were included, and 131 samples with strong fluorescence, 69 samples with vague fluorescence, and 67 samples with no fluorescence were collected. Tumor tissue was detected in all $131(100 \%)$ of the samples with strong fluorescence and in $65(94 \%)$ of the 69 samples with vague fluorescence. However, mostly infiltrative tumor tissue was still found in $33(49 \%)$ of 67 samples despite their lack of fluorescence. Strong fluorescence corresponded to compact tumors in 109 (83\%) of 131 samples, whereas vague fluorescence was consistent with infiltrative tumors in $44(64 \%)$ of 69 samples. In terms of the histopathological criteria of malignancy, a significant positive correlation of all analyzed parameters comprising cell density, nuclear pleomorphism, mitotic activity, microvascular proliferation, and necrosis with the 3 fluorescence levels was observed $(p<0.001)$. Furthermore, the proliferation rate significantly and positively correlated with strong (MIB-1 LI 28.3\%), vague (MIB-1 LI 16.7\%), and no (MIB-1 LI 8.8\%) fluorescence ( $p$ < 0.001). Last, a significantly higher microvessel density was detected in samples with strong fluorescence (CD34 125.5 vessels/0.25 mm²) than in those with vague (CD34 82.8 vessels $/ 0.25 \mathrm{~mm}^{2}$ ) or no (CD34 68.6 vessels $\left./ 0.25 \mathrm{~mm}^{2}\right)$ fluorescence $(p<0.001)$.

CONCLUSIONS Strong and vague 5-ALA-induced fluorescence enables visualization of intratumoral areas with specific histopathological features and thus supports neurosurgeons in improving the extent of resection in patients with a newly diagnosed GBM. Despite the lack of fluorescence, tumor tissue was still observed in approximately half of the

ABBREVIATIONS 5-ALA = 5-aminolevulinic acid; $C E$ = contrast enhancement; DTI = diffusion tensor imaging; fMRI = functional MRI; GBM = glioblastoma; IDH1 = isocitrate dehydrogenase $1 ; \mathrm{LI}=$ labeling index; NPV = negative predictive value; $\mathrm{PpIX}=$ protoporphyrin $\mathrm{IX} ; \mathrm{PPV}=$ positive predictive value.

SUBMITTED November 30, 2016. ACCEPTED April 4, 2017.

INCLUDE WHEN CITING Published online October 27, 2017; DOI: 10.3171/2017.4.JNS162991. 
cases. To overcome this current limitation, the promising approach of complementary spectroscopic measurement of fluorescence should be investigated further.

https://thejns.org/doi/abs/10.3171/2017.4.JNS162991

KEY WORDS glioblastoma; 5-ALA; fluorescence; histopathology; proliferation rate; microvessel density; oncology

$\mathrm{G}$ LIOBLASTOMA (GBM) constitutes the most frequent primary CNS tumor, accounting for approximately $45 \%-50 \%$ of all primary malignant brain tumors. ${ }^{17}$ This highly aggressive neoplasm is histopathologically characterized by a central necrosis surrounded by compact tumor tissue that transits into a zone of diffuse tumor infiltration of brain parenchyma. ${ }^{17,44}$ According to WHO criteria, typical histopathological features of GBM (WHO Grade IV) include increased cell density, nuclear pleomorphism, and mitotic activity as well as microvascular proliferation and necrosis. ${ }^{17}$ Moreover, GBM is characterized by a markedly increased proliferation rate and microvessel density. ${ }^{3,17,25}$ The maximum amount of these histopathological abnormalities is observed generally in the region of compact tumor tissue, but to a lesser extent, these features are present also in the area of infiltrative tumor tissue ${ }^{17}$ The standard treatment for the majority of patients suffering from a GBM consists of resection followed by concomitant radiotherapy and chemotherapy. ${ }^{5,23,36} \mathrm{With}$ regard to the extent of resection of the contrast-enhancing tumor, Sanai et al. ${ }^{28}$ recently found a stepwise survival benefit that starts at a resection rate of $78 \%$ of the contrast-enhancing tumor and reaches maximum survival advantage by achieving a $100 \%$ extent of resection. Accordingly, the goal of surgery in patients with a GBM is, whenever possible, the safe maximal removal of the contrast-enhancing tumor, which generally consists of compact tumor tissue. ${ }^{39}$ $\mathrm{Li}$ et al. ${ }^{16}$ recently found that also removing $\geq 53.21 \%$ of the surrounding FLAIR-depicted abnormality, which consists mainly of infiltrative tumor tissue, results in a further survival benefit. Thus, intraoperative identification of particular intratumoral histopathological regions is of major importance for achieving maximal GBM resection and, therefore, the best possible patient benefit. Nevertheless, these specific intratumoral areas of a GBM can frequently not be precisely identified by the neurosurgeon. As a consequence, it is hardly surprising that even complete resection of the contrast-enhancing tumor is achieved in only approximately $20 \%$ of patients with a GBM. ${ }^{1,19}$

To overcome this limitation, specific advancements, such as neuronavigation, ultrasound, and intraoperative MRI, have been introduced in past decades to improve intraoperative glioma identification. ${ }^{14,18,30,31}$ One of the most powerful techniques, however, is fluorescence-guided surgery, a real-time imaging method for intraoperatively visualizing GBM tissue independently of brain shift that only minimally interrupts surgical workflow. ${ }^{8,34,37}$ The most commonly used fluorescent agent is 5-aminolevulinic acid (5-ALA). ${ }^{8}$ In a multicenter Phase III trial in 2006, Stummer et al. ${ }^{33}$ demonstrated the superiority of 5-ALA fluorescence-guided surgery for GBM over conventional white-light procedures for achieving complete resection. Therefore, 5-ALA fluorescence-guided resection has been established at many neurosurgical departments world- wide as the current standard for surgery in patients with a GBM. ${ }^{8}$

In the course of 5-ALA fluorescence-guided surgery for GBM, the following 3 specific fluorescence levels usually can be distinguished within the same tumor by semiquantitative assessment: 1) strong fluorescence that histopathologically correlates predominantly to compact tumor, 2) vague fluorescence that corresponds primarily to infiltrative tumor, and 3) no fluorescence, commonly considered to denote absent or low infiltrative glioma tissue. . $^{8,13,34,35,40}$ However, a systematic histopathological analysis of tissues that show these 3 fluorescence levels still has not been performed, although 5-ALA fluorescence-guided surgery is widely used for GBM. Data from such a study would be of major importance for precisely guiding the neurosurgeon to identify particular intratumoral regions to maximize the rate of GBM resection.

Thus, the aim of our study was to systematically analyze tissue samples derived from 5-ALA fluorescenceguided GBM surgery according to relevant histopathological parameters. For this purpose, we prospectively collected tissue samples with strong, vague, or no 5-ALAinduced fluorescence in a large series of newly diagnosed GBMs. Each sample was analyzed for the presence of tumor tissue, the quality of tissue (compact, infiltrative, or no tumor), histopathological criteria of malignancy (cell density, nuclear pleomorphism, mitotic activity, microvascular proliferation, and necrosis), proliferation rate (MIB-1 labeling index [LI]), and microvessel density (using CD34 staining).

\section{Methods}

Between 2008 and 2013, patients who underwent 5-ALA fluorescence-guided resection of a radiologically suspected newly diagnosed GBM at the Medical University of Vienna Department of Neurosurgery were prospectively recruited. This study was approved by the local ethics committee of the Medical University of Vienna (EK 419/2008/Amendment), and informed consent was given by each patient.

\section{Study Cohort: Inclusion and Exclusion Criteria}

Adult patients (aged $\geq 18$ years) who underwent 5-ALA fluorescence-guided resection of a radiologically suspected newly diagnosed supratentorial GBM with characteristic ring-like contrast-enhancement (CE) found on preoperative MRI were recruited. Thus, patients with a known or surgically treated WHO Grade II or III glioma, recurrent GBM, history of chemotherapy and/or radiotherapy for a glioma, biopsy only, or infratentorial tumor localization were excluded from this study. Patients with known contraindications for 5-ALA administration were excluded, as described elsewhere. ${ }^{33}$ To enable histopathological analysis of a uniform and by far the most common glioma 
subgroup, only patients with a postsurgical histopathological diagnosis of a GBM (WHO Grade IV) were included, and thus these patients formed our final study cohort.

\section{Preoperative Imaging}

In each patient, a diagnostic MRI study of the brain including contrast medium administration was conducted within 2 weeks before tumor resection. Tumor localization and the pattern of CE on MRI (ring-like, nodular, focal, patchy/faint, or none) were determined in each patient as described previously by an experienced neuroradiologist (A.M.). ${ }^{41-43}$ According to our inclusion criteria, only patients with a radiologically suspected GBM with ringlike CE were included in this study. After hospitalization, further contrast-enhanced T1-weighted images were obtained for integration of these image data into the navigation system. Depending on the tumor localization, additional diffusion tensor imaging (DTI) and/or functional MRI (fMRI) was conducted.

\section{5-ALA Fluorescence-Guided GBM Resection}

A standard dose of 5-ALA (20 mg/kg of body weight) was administered orally approximately 3 hours before surgery for each patient ${ }^{37,40}$ For intraoperative visualization of 5-ALA-induced protoporphyrin IX (PpIX) fluorescence, we used an adapted neurosurgical microscope (NC4 or Pentero, Carl Zeiss Surgical GmbH) capable of switching to violet-blue excitation light..$^{34,37,40}$ To avoid potential 5-ALA-related phototoxicity, each patient was protected from strong light sources for a minimum time period of 24 hours after 5-ALA administration. During surgery, we routinely used a navigation system (StealthStation S7 or Treon, Medtronic) with integrated contrast-enhanced T1weighted sequences for precise localization of the GBM and intraoperative guidance. Furthermore, GBM resection was performed with the assistance of 5-ALA fluorescence guidance. Depending on the localization of the tumor, preoperatively acquired DTI and/or fMRI data integrated into the navigation system and/or intraoperative mapping and stimulation were used also.

\section{Intraoperative Tissue Sampling}

The adapted microscope was switched repetitively to violet-blue excitation light during tumor resection to visualize the following 5-ALA-induced fluorescence levels that usually can be distinguished in GBMs: 1) strong fluorescence, indicated by an intensive red fluorescing pattern, 2) vague fluorescence, which has a less intensive salmoncolored fluorescing appearance, and 3) no fluorescence, which has a nonfluorescing blue quality. ${ }^{8,13,32,35,40}$ During surgery, the maximum fluorescence level (strong, vague, or none) in each tumor was determined by a neurosurgeon experienced with regard to 5-ALA fluorescence-guided procedures. Then, 1 or more tissue samples were collected from this fluorescence level for histopathological correlation. Moreover, during the surgery, additional tissue samples from areas with vague and/or no fluorescence subsequent to fluorescing intratumoral regions were taken for histopathological analysis, if it was safe to do so without risking a neurological deficit.

\section{Assessment of Interobserver Agreement on Fluorescence-Level Classification}

Because the semiquantitative assessment of these 3 fluorescence levels by the neurosurgeon is subjective, we also investigated the interobserver agreement of our fluorescence classifications. To that end, we created a file with intraoperative photographs of various fluorescence levels $(n=45)$ derived from 5-ALA fluorescence-guided resections of newly diagnosed GBMs at our department. We then asked 6 neurosurgeons in our department who were experienced in 5-ALA fluorescence-guided procedures to separately classify the different fluorescence levels of these intraoperative photographs as strong, vague, or none. Each neurosurgeon reviewed the same 45 intraoperative photographs of the various fluorescence levels.

\section{Histopathology}

Tumor Diagnosis

The resected tumor bulk and all collected tissue samples from each patient were formalin fixed, paraffin embedded, and processed for routine histopathological/ immunohistochemical staining. In each patient, the histopathological tumor diagnosis was established by the local neuropathology team according to the 2016 update of the WHO Classification of Tumours of the Central Nervous System. ${ }^{17}$ In this sense, the isocitrate dehydrogenase (IDH1)-R132H mutation-specific antibody (anti-human IDH1-R132H, 1:30 at pH6 [Dianova]) was applied to assess the IDH1 mutational status.

\section{Analysis of Fluorescing and Nonfluorescing Tissue Samples}

In addition, all collected fluorescing and nonfluorescing tissue samples were histopathologically analyzed by 2 experienced neuropathologists (J.A.H. and A.W.) who were blinded to the intraoperative fluorescence status. For that analysis, we used conventional histopathological staining ( $\mathrm{H} \& \mathrm{E}$ ) and immunohistochemistry to assess the proliferation rate and microvessel density. Immunohistochemistry analyses were conducted using an automated slide staining system (BenchMark Ultra, Ventana Medical Systems, Inc.).

In our analysis, the following parameters were assessed in each tissue sample.

Presence of Tumor Tissue. All collected tissue samples were screened for the presence of tumor tissue using standard H \& E staining.

Quality of Tissue. In the next step, the tissue of each sample was further characterized and classified as compact, infiltrative, or having no tumor. In this study, compact tumors were defined as having densely packed tumor cells, whereas infiltrative tumors were considered to be loosely arranged pleomorphic tumor cells intermingled with preexisting brain parenchyma.

Histopathological Criteria of Malignancy. Predefined histopathological criteria of malignancy according to the WHO were analyzed for each tissue sample using a modified semiquantitative grading system ${ }^{41,42}$ that considers cell density (normal, low, moderate, or high), nuclear pleomorphism (normal, low, moderate, or high), mitotic activity 
(absent, few, some, or many), presence of microvascular proliferation (yes or no), and necrosis (yes or no).

Proliferation Rate. The proliferation rate in each tissue sample was measured at hot spots and documented as the percentage of immunolabeled cells using the Ki-67 antibody (MIB-1 LI, 1:50 at pH 6 [Dako, Glostrup, Denmark]) as described previously. ${ }^{41-43}$

Microvessel Density. The microvessel density was analyzed by counting CD34-immunolabeled vessels (antiCD34, Novocastra [Leica Biosystems Newcastle Ltd]) per defined areas of $0.25 \mathrm{~mm}^{2}$ at hot spots of greatest vascular density, as described elsewhere. ${ }^{24}$

\section{Postoperative Outcome}

The neurological status of each patient was routinely assessed before surgery, at the time of discharge from the neurosurgical department, and at a 3-month followup visit. A neurological deficit was considered transient if it resolved completely within 3 months after surgery and permanent if it was still present 3 months after surgery.

\section{Statistical Analysis}

Statistical analysis was conducted using SPSS 23.0 software (SPSS, Inc.). To assess interobserver agreement on classification of the 3 fluorescence levels, we applied the Cohen kappa test. Interpretation of kappa values was performed as previously described ${ }^{11,22}(<0.2$, poor interobserver agreement; 0.2-0.4, slight interobserver agreement; $0.4-0.6$, moderate interobserver agreement; $0.6-0.8$, substantial interobserver agreement; $0.8-1$, almost perfect interobserver agreement). The Kendall-tau-b correlation coefficient was applied for correlation of the 5-ALA-induced fluorescence levels (strong, vague, and no fluorescence) with the presence of tumor tissue, quality of tissue, histopathological criteria of malignancy, MIB-1 LI, and microvessel density. For correlation of the fluorescence levels with MIB-1 LI and microvessel density, ANOVA was performed. In case of a significant difference according to the ANOVA, subsequent testing for differences between specific fluorescence levels (strong vs vague fluorescence, strong vs no fluorescence, and vague vs no fluorescence) was performed by using unpaired t-tests. Furthermore, specificity, sensitivity, positive predictive value (PPV), and negative predictive value (NPV) for visible fluorescence and tumor tissue-containing samples and the $95 \%$ confidence interval were calculated. A p value of $<0.05$ was considered significant.

\section{Results}

Altogether, 83 adult patients with 5-ALA fluorescenceguided resection of a radiologically suspected newly diagnosed supratentorial GBM were included in this study initially. Of these patients, 6 had to be excluded because their postsurgical histopathological diagnosis was not GBM (3 lymphomas, 2 metastases, and 1 WHO Grade III anaplastic glioma). Therefore, 77 patients with a histopathologically confirmed newly diagnosed GBM remained and formed our final study cohort. In all of these patients, tissue sampling from different fluorescing and/or nonfluo- rescing areas of the newly diagnosed GBM for histopathological analysis was feasible in the course of their 5-ALA fluorescence-guided resection. In none of these patients did relevant 5-ALA-associated adverse effects occur.

\section{Study Cohort}

The median age of the 77 patients (46 male and 31 female) was 60 years (range 27-82 years). An IDH1-R132H mutation was detected by immunohistochemistry in 4 (5\%) of the 77 patients. The most common tumor localizations were the frontal (17 [22\%] patients), temporal (16 [21\%] patients), and parietal (11 [14\%] patients) lobes. In 7 patients $(9 \%)$, a multifocal $(n=5)$ or multicentric $(n=2)$ tumor localization was present. Additional details regarding the patient cohort are provided in Table 1.

\section{5-ALA Fluorescence-Guided GBM Resection}

We observed 5-ALA-induced fluorescence in every GBM in the course of tumor resection. Strong fluorescence was found to be the maximum level in all but 1 patient (76 [99\%] patients). In this 1 patient (1\%), only vague fluorescence was detected. In tumors that showed both strong and vague fluorescence (76 [99\%] patients), intratumoral areas with strong fluorescence were usually

TABLE 1. Patients characteristics

\begin{tabular}{|c|c|}
\hline Characteristic & Value \\
\hline No. $(\%)$ of patients & $77(100)$ \\
\hline Sex (female/male) & $1: 1.48$ \\
\hline Age (yrs) (median [range]) & $60(27-82)$ \\
\hline \multicolumn{2}{|l|}{ KPS score (no. [\%]) } \\
\hline $100 \%$ & $9(12)$ \\
\hline $90 \%$ & $34(44)$ \\
\hline $80 \%$ & $31(40)$ \\
\hline$\leq 70 \%$ & $3(4)$ \\
\hline \multicolumn{2}{|l|}{ Localization (no. [\%]) } \\
\hline Frontal & $17(22)$ \\
\hline Temporal & $16(21)$ \\
\hline Parietal & $11(14)$ \\
\hline Trigonal & $9(12)$ \\
\hline Multifocal/multicentric & $7(9)$ \\
\hline Occipital & $7(9)$ \\
\hline Insular & $4(5)$ \\
\hline Limbic & $3(4)$ \\
\hline Central & $2(3)$ \\
\hline Corpus callosum & $1(1)$ \\
\hline \multicolumn{2}{|c|}{ Maximal fluorescence status (no. [\%]) } \\
\hline Strong & $76(99)$ \\
\hline Vague & $1(1)$ \\
\hline None & $0(0)$ \\
\hline \multicolumn{2}{|c|}{ IDH1-R132H mutation (no. [\%]) } \\
\hline No & $73(95)$ \\
\hline Yes & $4(5)$ \\
\hline
\end{tabular}

KPS = Karnofsky Performance Scale. 
surrounded by tumor regions with vague fluorescence. In total, 267 tissue samples were collected from the 77 patients during fluorescence-guided surgery (median 3 per patient; range 1-11 per patient). Of these tissue samples, 200 revealed visible fluorescence (131 samples with strong fluorescence, 69 samples with vague fluorescence). In contrast, no fluorescence was found in the remaining 67 samples. For further details see Tables 1 and 2.

\section{Interobserver Agreement on Fluorescence-Level Classification}

To assess the interobserver agreement on our classification of fluorescence, 6 neurosurgeons separately classified various fluorescence levels in the same 45 intraoperative photographs derived from the resection of newly diagnosed GBMs as strong, vague, or none. According to our data, we had almost perfect interobserver agreement among these neurosurgeons (kappa value range $0.83-1$ ) (Fig. 1).

\section{Histopathological Analysis}

All 267 tissue samples with strong, vague, or no fluorescence were analyzed systematically for the presence of tumor tissue, quality of tissue, histopathological criteria of malignancy, proliferation rate, and microvessel density. Detailed overviews are provided in Table 2 and Figs. 2 and 3.

\section{Strong Fluorescence}

Tumor tissue was detected in all (131 [100\%]) samples with strong fluorescence, and this tissue corresponded to compact tumor in the majority (109 [83\%] of 131) of samples. Analysis according to the histopathological criteria

TABLE 2. Systematic histopathological analysis of 267 tissue samples with strong, vague, or no fluorescence

\begin{tabular}{|c|c|c|c|c|c|}
\hline \multirow[b]{2}{*}{ Parameter } & \multicolumn{3}{|c|}{ 5-ALA-Induced Fluorescence* } & \multirow[b]{2}{*}{$p$ Value } & \multirow[b]{2}{*}{$\mathrm{CC}$} \\
\hline & Strong $(n=131)$ & Vague $(n=69)$ & None $(n=67)$ & & \\
\hline \multicolumn{4}{|l|}{ Tumor tissue } & \multirow{3}{*}{$<0.001$} & \multirow{3}{*}{0.51} \\
\hline Yes & $131(100)$ & $65(94)$ & $33(49)$ & & \\
\hline No & $0(0)$ & $4(6)$ & $34(51)$ & & \\
\hline Quality of tissue & & & & $<0.001$ & 0.70 \\
\hline Compact tumor & $109(83)$ & $21(30)$ & $1(1)$ & & \\
\hline Infiltrative tumor & $22(17)$ & $44(64)$ & $32(48)$ & & \\
\hline No tumor & $0(0)$ & $4(6)$ & $34(51)$ & & \\
\hline Cell density & & & & $<0.001$ & 0.63 \\
\hline Normal & $0(0)$ & $4(6)$ & $34(51)$ & & \\
\hline Low & $7(5)$ & $25(36)$ & $16(24)$ & & \\
\hline Moderate & $43(33)$ & $28(41)$ & $15(22)$ & & \\
\hline High & $81(62)$ & $12(17)$ & $2(3)$ & & \\
\hline \multicolumn{4}{|l|}{ Nuclear pleomorphism } & \multirow{5}{*}{$<0.001$} & \multirow{5}{*}{0.62} \\
\hline Normal & $0(0)$ & $4(6)$ & $34(50)$ & & \\
\hline Low & $5(4)$ & $21(30)$ & $13(20)$ & & \\
\hline Moderate & $25(19)$ & $23(34)$ & $13(20)$ & & \\
\hline High & $101(77)$ & $21(30)$ & $7(10)$ & & \\
\hline \multicolumn{4}{|l|}{ Mitotic activity } & \multirow{5}{*}{$<0.001$} & \multirow{5}{*}{0.55} \\
\hline Absent & $0(0)$ & $4(6)$ & $34(51)$ & & \\
\hline Few & $18(14)$ & $39(57)$ & $17(25)$ & & \\
\hline Some & $50(38)$ & $18(26)$ & $9(13)$ & & \\
\hline Many & $63(48)$ & $8(12)$ & $7(10)$ & & \\
\hline \multicolumn{4}{|l|}{ Microvascular proliferation } & \multirow{3}{*}{$<0.001$} & \multirow{3}{*}{0.59} \\
\hline Yes & $125(95)$ & $50(72)$ & $16(24)$ & & \\
\hline No & $6(5)$ & $19(28)$ & $51(76)$ & & \\
\hline \multicolumn{4}{|l|}{ Necrosis } & \multirow{3}{*}{$<0.001$} & \multirow{3}{*}{0.55} \\
\hline Yes & $100(76)$ & $24(35)$ & $5(7)$ & & \\
\hline No & $31(24)$ & $45(65)$ & $62(93)$ & & \\
\hline MIB-1 LI (\%) (mean \pm SD) & $28.3 \pm 13.2$ & $16.7 \pm 12.1$ & $8.8 \pm 10.8$ & $<0.001$ & 0.47 \\
\hline CD34 (vessels/0.25 mm²) (mean \pm SD) & $125.5 \pm 63.5$ & $82.8 \pm 47.1$ & $68.6 \pm 36.8$ & $<0.001$ & 0.33 \\
\hline
\end{tabular}


of malignancy revealed that samples with strong fluorescence were characterized predominantly by high cell density (81 [62\%] of 131), nuclear pleomorphism (101 [77\%] of 131), and some to many mitotic figures (113 [86\%] of 131). Furthermore, microvascular proliferation (125 [95\%] of 131) and focal necrosis (100 [76\%] of 131) were the predominant features in most tissue samples. Also, samples with strong fluorescence revealed a mean proliferation rate of $28.3 \%( \pm 13.2 \%)$ and microvessel density of 125.5 vessels $/ 0.25 \mathrm{~mm}^{2}\left( \pm 63.5\right.$ vessels $\left./ 0.25 \mathrm{~mm}^{2}\right)$.

\section{Vague Fluorescence}

Tumor tissue was identified in the vast majority of samples with vague fluorescence (65 [94\%] of 69), and this tissue corresponded mainly to infiltrative tumor (44 [64\%] of 69). Samples with vague fluorescence were characterized predominantly by low-to-moderate cell density (53 [77\%] of 69), low-to-high nuclear pleomorphism (65 [94\%] of 69), and few mitotic figures (39 [57\%] of 69). Moreover, microvascular proliferation was present (50 [72\%] of 69) but focal necrosis was absent (45 [65\%] of 69 samples) in most tissue samples. Also, samples with vague fluorescence revealed a mean proliferation rate of $16.7 \%( \pm$ $12.1 \%$ ) and microvessel density of 82.8 vessels $/ 0.25 \mathrm{~mm}^{2}$ $\left( \pm 47.1\right.$ vessels $/ 0.25 \mathrm{~mm}^{2}$ ).

\section{No Fluorescence}

Tumor tissue was present in approximately half of the samples with no fluorescence (33 [49\%] of 67), and this tissue was composed almost exclusively of infiltrative tumor (32 [97\%] of 33). In contrast, no distinct tumor cells were found in the other half of the samples (34 [51\%] of 67). Samples with no fluorescence were characterized predominantly by normal-to-moderate cell density (65 [97\%] of 67), nuclear pleomorphism (60 [90\%] of 67), and no to few mitotic figures (51 [76\%] of 67). Moreover, an absence of microvascular proliferation (51 [76\%] of 67) and focal necrosis (62 [93\%] of 67) was observed in the majority of samples. Also, samples with no fluorescence revealed a mean proliferation rate of $8.8 \%( \pm 10.8 \%)$ and microvessel density of 68.6 vessels $/ 0.25 \mathrm{~mm}^{2}( \pm 36.8$ vessels $/ 0.25$ $\left.\mathrm{mm}^{2}\right)$.

\section{Correlation of Histopathology With Specific Fluorescence Levels}

First, we found that presence of tumor, quality of tissue, and all investigated histopathological criteria of malignancy, including cell density, nuclear pleomorphism, mitotic activity, microvascular proliferation, and necrosis, correlated significantly with the fluorescence levels (strong, vague, or no fluorescence; $\mathrm{p}<0.001)$. Furthermore, we observed that the proliferation rate correlates significantly with the fluorescence levels $(p<0.001)$. Also, a significantly higher microvessel density was detected in samples with strong fluorescence than in those with vague or no fluorescence $(\mathrm{p}<0.001)$. However, no significant difference in terms of microvessel densities was found in the samples with vague fluorescence and in those with no fluorescence $(p=0.053)$.

\section{Samples With False-Positive Fluorescence}

In $4(2 \%)$ of 200 fluorescing tissue specimens, no dis-

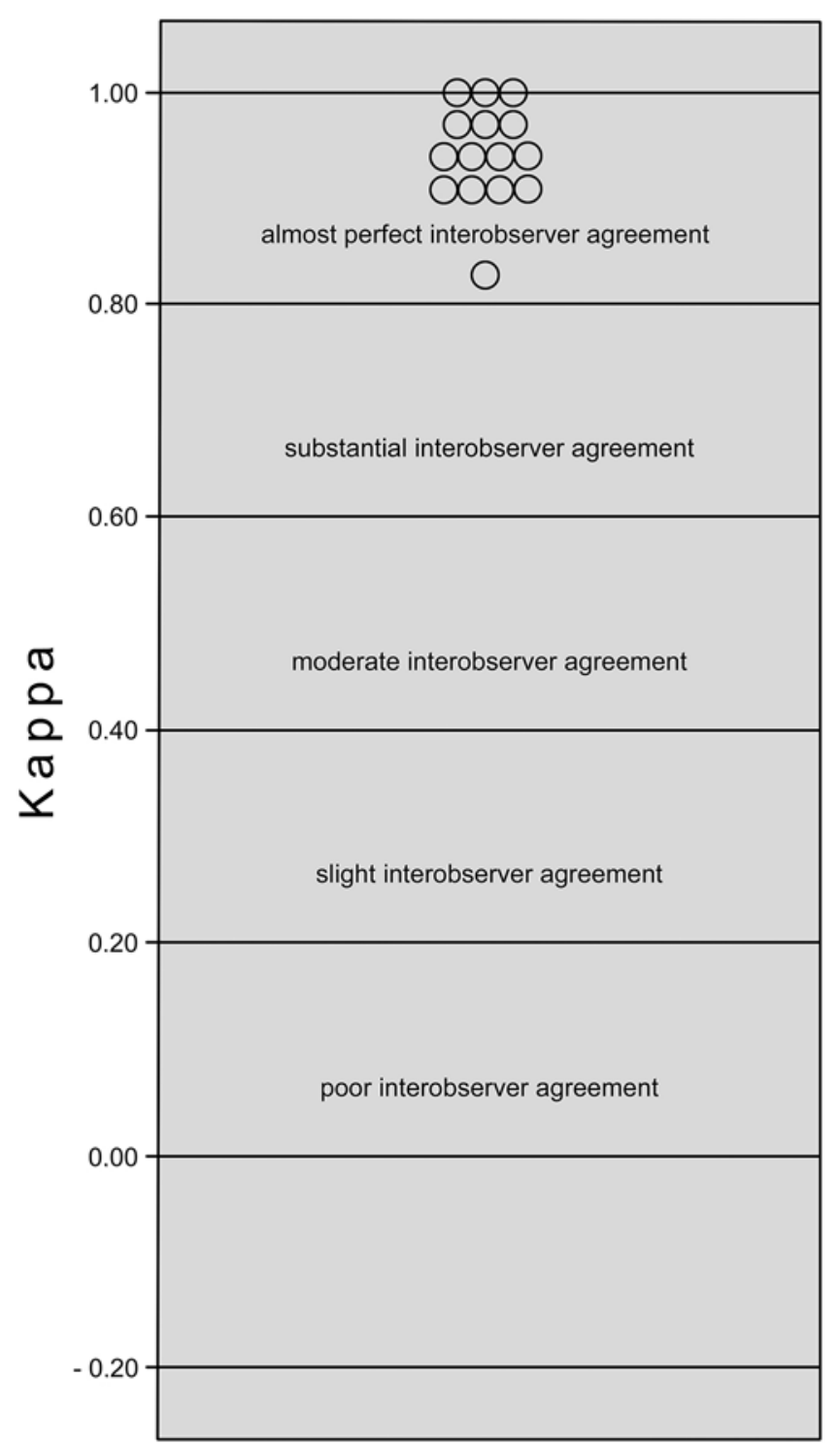

FIG. 1. Illustration of the interobserver agreement on the classification of the three 5-ALA-induced fluorescence levels according to kappa values. The various fluorescence levels on the same 45 intraoperative photographs derived from resections of newly diagnosed GBMs were classified separately by 6 neurosurgeons as strong, vague, or none. The analysis of these data revealed that all the kappa values were in the range of $0.83-1$. As a consequence, we found almost perfect interobserver agreement among these neurosurgeons.

tinct tumor cells were found with conventional histopathology, although these samples had visible fluorescence. False-positive fluorescence was observed only in tissue samples with vague fluorescence, never in samples with strong fluorescence. Although no distinct tumor cells were detected in these 4 tissue samples with vague fluorescence, reactive astrocytes were observed in all of them. In addition, in 2 of these samples, endothelial cell activation was found.

\section{Sensitivity, Specificity, PPV, and NPV}

In further analysis, the sensitivity, specificity, PPV, and NPV of visible fluorescence for the detection of tumor tis- 

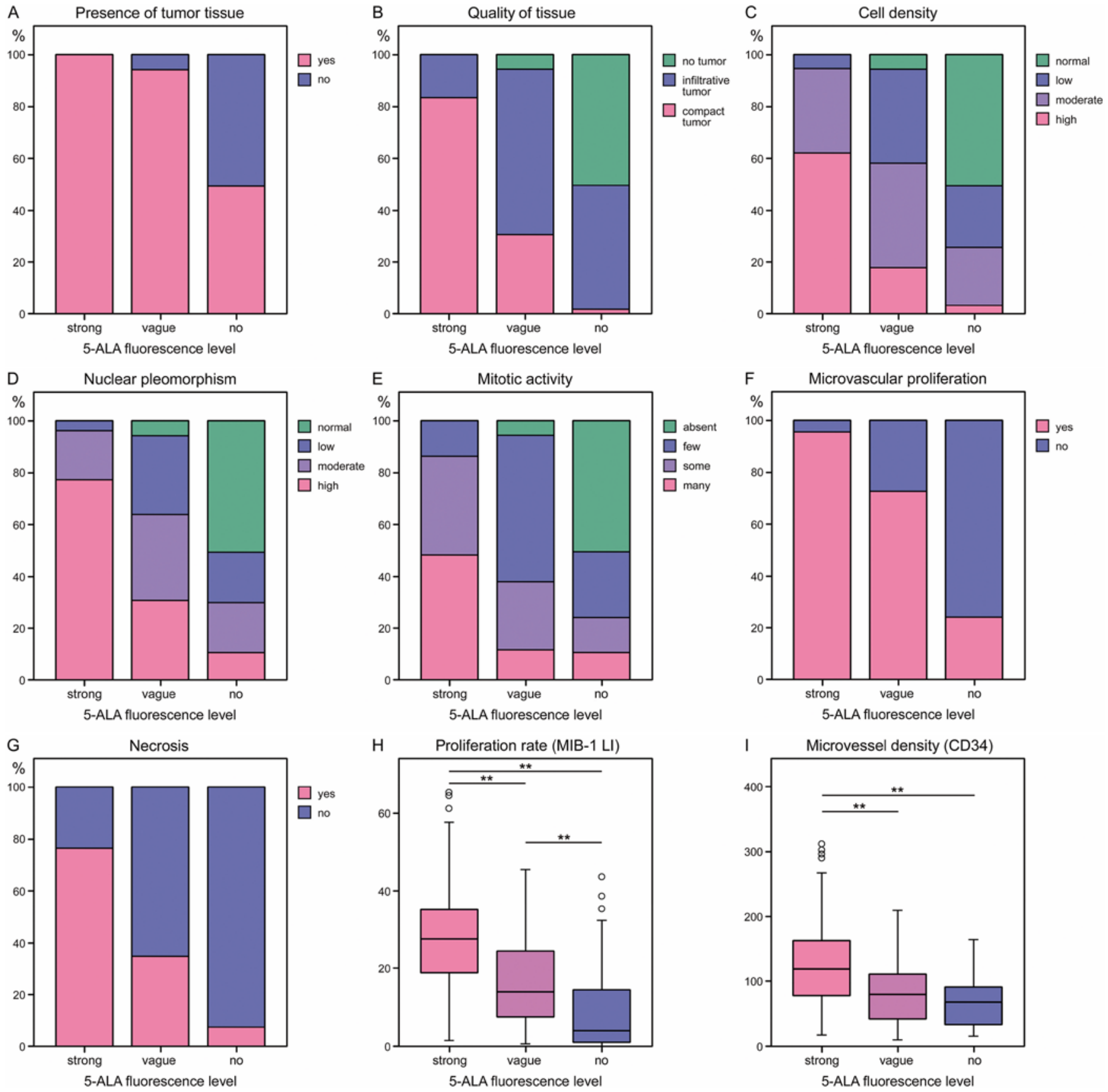

FIG. 2. Boxplot diagrams showing the distribution of relevant histopathological parameters in samples with strong, vague, or no fluorescence. A: Tumor tissue was present in 100\% of samples with strong fluorescence, $94 \%$ with vague fluorescence, and $49 \%$ with no fluorescence. B: Compact tumor was observed in $83 \%$ of samples with strong fluorescence, whereas $64 \%$ of specimens with vague fluorescence were composed of infiltrative tumor. The half of the samples with no fluorescence-containing tumor tissue were in $97 \%$ of cases composed of infiltrative tumor. C: $62 \%$ of the samples that showed strong fluorescence had high cell density, $77 \%$ of those that showed vague fluorescence had low-to-moderate cell density, and $97 \%$ of those that showed no fluorescence had normal-to-moderate cell density. D: In $77 \%$ of samples with strong fluorescence, high nuclear pleomorphism was found, in $94 \%$ of samples with vague fluorescence, low-to-high nuclear pleomorphism was found, and in $90 \%$ of the samples with no fluorescence, normal-to-moderate nuclear pleomorphism was found. E: In $86 \%$ of samples with strong fluorescence, some to many mitotic figures were present, in $57 \%$ of samples with vague fluorescence, few mitotic figures were present, and in $76 \%$ of samples with no fluorescence, no to few mitotic figures were present. F: Microvascular proliferation was detected in $95 \%, 72 \%$, and $24 \%$ of samples with strong, vague, or no fluorescence, respectively. G: Necrotic foci were found in $76 \%, 35 \%$, and $7 \%$ of samples with strong, vague, or no fluorescence, respectively. $\mathrm{H}$ : A significant correlation of the proliferation rate with the fluorescence levels was present (MIB-1 LI 28.3\%, 16.7\%, and 8.8\% in samples with strong, vague, or no fluorescence, respectively). ${ }^{* *} p<0.001$. I: Significantly higher microvessel density was detected in samples with strong fluorescence (CD34 125.5 vessels $/ 0.25 \mathrm{~mm}^{2}$ ) than in those with vague (CD34 82.8 vessels $\left./ 0.25 \mathrm{~mm}^{2}\right)$ or no (CD34 68.6 vessels $\left./ 0.25 \mathrm{~mm}^{2}\right)$ fluorescence $\left({ }^{* *} \mathrm{p}<0.001\right)$. However, the difference in microvessel densities in samples with vague and in those with no fluorescence did not reach statistical significance $(p=0.053)$. 

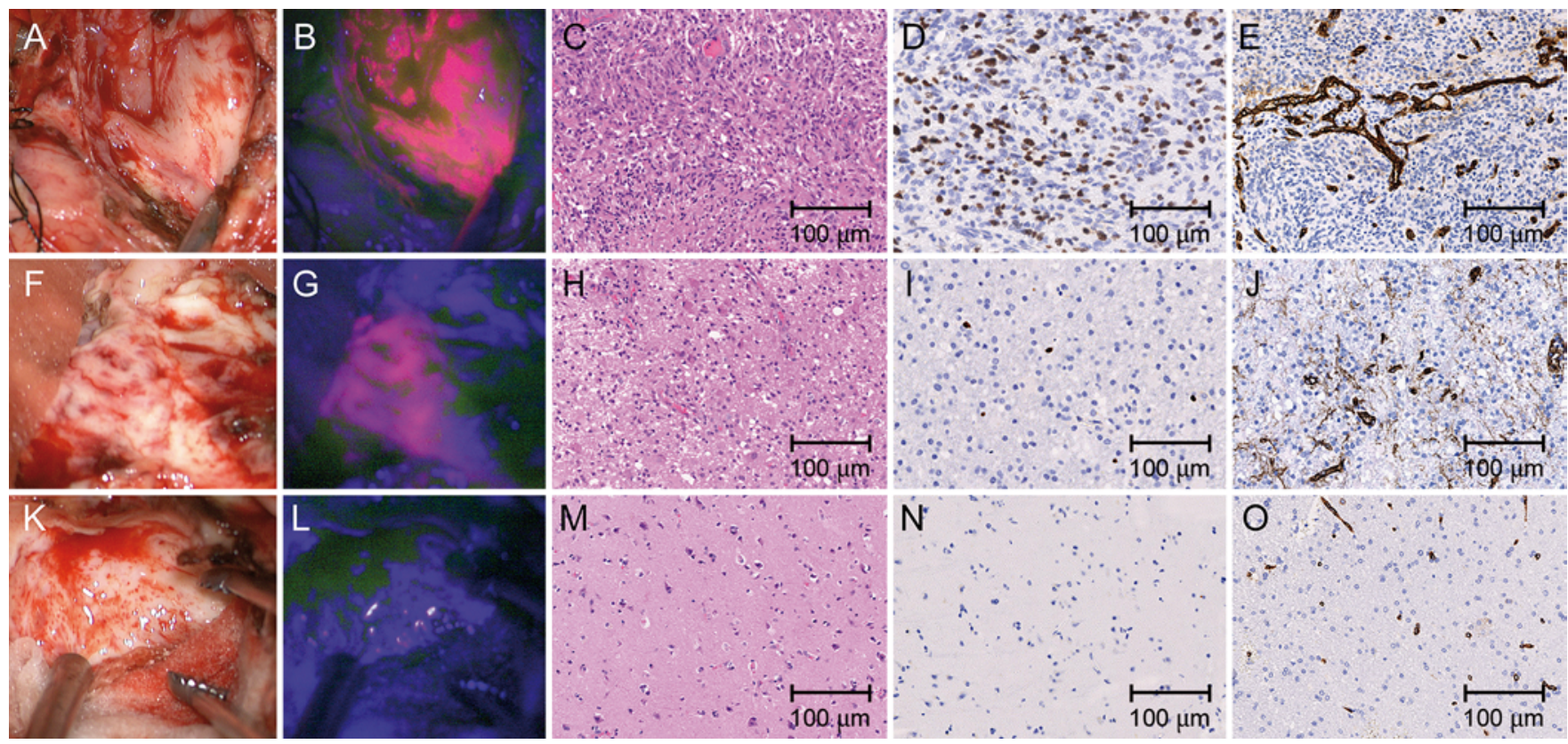

FIG. 3. Representative patient with a newly diagnosed GBM in the right frontal lobe: correlation of intraoperative conventional white-light microscopy and 5-ALA-induced fluorescence levels with corresponding histopathology, proliferation rate, and microvessel density. At the beginning of surgery, an intratumoral region with distinct tumor tissue under conventional white-light microscopy $(\mathbf{A})$ shows strong fluorescence after excitation with violet-blue light (B). The corresponding histopathology reveals the presence of compact tumor with high cell density, nuclear pleomorphism, and mitotic activity and the presence of microvascular proliferation and necrosis (C) with a markedly increased proliferation rate (D) and high microvessel density (E). In the course of surgery, another intratumoral region reveals only minor pathological changes under white-light microscopy (F) but shows vague fluorescence under violet-blue excitation light $(\mathbf{G})$. The corresponding histopathology shows the presence of infiltrative tumor with moderate cell density, nuclear pleomorphism, low mitotic activity, and presence of microvascular proliferation but an absence of necrosis $(\mathrm{H})$ and a moderately increased proliferation rate $(\mathrm{I})$ and microvessel density $(\mathrm{J})$. At the end of surgery, an intratumoral area at the assumed margin of the tumor infiltration zone and directly subsequent to a region previously showing vague fluorescence shows no distinct tumor tissue under white-light microscopy $(\mathbf{K})$, and no fluorescence is visible $(\mathbf{L})$. The corresponding histopathology shows reactive changes but no definite tumor tissue with normal cell density and nuclear pleomorphism, absent mitotic activity, microvascular proliferation, and necrosis $(\mathbf{M})$ without cell proliferation $(\mathbf{N})$ and with normal microvessel density $(\mathbf{0})$.

sue were calculated. According to our data, the sensitivity and specificity were $86 \%$ and $89 \%$, respectively. In addition, the PPV was $98 \%$ (strong fluorescence $100 \%$, vague fluorescence 94\%), and the NPV was 51\%. Additional details are provided in Table 3.

\section{Postoperative Neurological Outcome}

In none of the 77 patients included in this study did a perioperative death occur. Postoperative deterioration of preexisting neurological deficits at the time of discharge was observed in $2(3 \%)$ of 77 patients (a permanent worsening of hemiparesis in 1 patient and organic psychosyndrome in the other patient). New postoperative neurological deficits were found in $4(5 \%)$ of 77 patients (transient oculomotor palsy, transient aphasia, permanent hemiparesis, and permanent aphasia/hemiparesis in 1 patient each). The main causes for these neurological deficits were a space-occupying postsurgical hemorrhage in 3 patients and postoperative swelling, ischemia, and surgical manipulation in 1 patient.

\section{Discussion}

In this study, we performed a systematic histopathological analysis of tissue samples derived during 5-ALA fluorescence-guided surgery with strong, vague, or no fluorescence in 77 patients with a newly diagnosed supratentorial GBM. According to our data, strong and vague fluorescence is clinically reliable for visualizing intratumoral areas with specific histopathological features. In this sense, strong fluorescence supports the neurosurgeon in identifying compact tumors, whereas vague fluorescence is useful for visualizing tissue that consists predominantly of infiltrative tumor to improve the extent of resection. However, mainly infiltrative tumor tissue was still present in approximately half of our patients despite the lack of fluorescence; thus, complementary tools such as spectroscopic measurement of fluorescence should be investigated to overcome this current limitation of 5-ALA.

\section{5-ALA in GBM: Lack of a Systematic Histopathological Analysis}

Intraoperative visualization of GBM tissue with the assistance of 5-ALA-induced fluorescence represents an innovative and widely used real-time imaging method that is independent of brain shift and results in only minimal interruption of surgical workflow. ${ }^{8,34,37}$ During 5-ALA fluorescence-guided surgery in patients with a GBM, 3 characteristic fluorescence levels (strong, vague, 
TABLE 3. Predictive relationship of visible 5-ALA-induced fluorescence and tumor tissue-containing specimens

\begin{tabular}{lc}
\hline \multicolumn{1}{c}{ Factor } & Specimens w/ Tumor Tissue $(\%[95 \% \mathrm{Cl}])$ \\
\hline Sensitivity & $86(80-90)$ \\
\hline Specificity & $89(76-96)$ \\
\hline PPV & $98(95-99)$ \\
\hline Strong fluorescence & $100(97-100)$ \\
\hline Vague fluorescence & $94(86-98)$ \\
\hline NPV & $51(39-62)$ \\
\hline
\end{tabular}

and none) usually can be observed. . $^{8,13,22,35,40}$ These specific fluorescence levels also can be detected by spectrometric assessment and show significant differences in the characteristic 5-ALA-induced PpIX peak. ${ }^{35}$ Although the application of $4^{13,15,27}$ or even $5^{4,13,38}$ fluorescence levels in malignant gliomas was described by some groups in past years, the 3-fluorescence-level classification system is by far the most widely used other than dichotomized categorization systems (presence or absence of fluorescence only), according to a recently published systematic literature review. ${ }^{13}$ So far, however, the exact histopathological correlate of these 3 characteristic fluorescence levels, and thus their surgical significance for visualization of specific intratumoral areas in GBMs, has not been clarified sufficiently. Important reasons for this limitation found in other studies include the following: 1) GBMs were not analyzed separately but, rather, together with gliomas of different WHO grades, ${ }^{8,10,15}$ 2) recurrent gliomas in which previous radiotherapy and/or chemotherapy might have resulted in reactive tissue alterations were included, and thus potential false-positive fluorescence might have occurred, ${ }^{6-8,12,15} 3$ ) more than 3 fluorescence levels were applied, 4,13,15,27,38 and 4) mostly only single histopathological parameters that were focused mainly on the quality of tissue and cell density were investigated..$^{6,15,32,35}$

\section{Current Study: Systematic Histopathological Analysis of 5-ALA Fluorescence in GBMs}

We designed the current prospective study and included in the final analysis only patients with a histopathologically confirmed newly diagnosed GBM to avoid potential treatment-related influence on intraoperative fluorescence. From this large series of 77 patients, 267 tissue samples with strong, vague, or no fluorescence were collected for systematic histopathological assessment. In each of these tissue samples, we analyzed separately the following relevant histopathological parameters: 1) presence of tumor, 2) quality of tissue, 3) histopathological criteria of malignancy, 4) proliferation rate, and 5) microvessel density.

According to the data of this study, we typically observed the following histopathological features in tissue samples with the 3 different fluorescence levels.

\section{Presence of Tumor Tissue}

As a precursor of heme biosynthesis, 5-ALA results, after oral administration, in the accumulation of fluorescing PpIX in malignant glioma cells; thus, tumor tissue of GBMs can be visualized intraoperatively by fluo- rescence. ${ }^{8,34}$ In this sense, we detected tumor tissue in all samples with strong fluorescence and in the majority of samples with vague fluorescence (94\%). As a consequence, we found a very high PPV of both strong $(100 \%)$ and vague (94\%) fluorescence for the presence of tumor tissue. These results are in accordance with those of a study published recently by Stummer et al., ${ }^{35}$ in which a PPV of $100 \%$ for samples with strong fluorescence and a PPV of $91 \%$ for samples with vague fluorescence were found in a separate analysis that included only newly diagnosed GBMs.

In contrast, in our study, samples with no fluorescence had an absence of tumor tissue in only approximately half of the cases, resulting in an NPV of $51 \%$. One possible explanation for this relatively low NPV might be that a slight infiltration with tumor cells can typically be detected in GBMs even several centimeters apart from the tumor bulk. Another explanation might be that the 5-ALA-induced PpIX accumulation is too low to induce visible fluorescence in areas with such slight tumor infiltration. The NPV of 5-ALA-induced fluorescence strongly depends on the site of tissue collection and, thus, the distance from the tumor bulk. ${ }^{8,35}$ Therefore, it is hardly surprising that the reported NPVs of 5-ALA-induced fluorescence in the literature in newly diagnosed GBMs have had a relatively wide range. Panciani et al..$^{20,21}$ observed in their 2 studies very high NPVs (approximately 90\%), whereas Roberts et al. ${ }^{27}$ and Stummer et al. ${ }^{35}$ found considerably lower NPVs $(26 \%$ and $40 \%$, respectively). In our study, nonfluorescing samples were collected subsequent to intratumoral areas with visible fluorescence within the region of the suspected tumor margin, whereas in other studies, tissue sampling was performed also from adjacent ("distant") brain regions. ${ }^{35}$

\section{Quality of Tissue}

In further characterization of the quality of tissue of each sample, we observed in our study that samples with strong fluorescence corresponded to compact tumor in the majority of cases (83\%), whereas specimens with vague fluorescence were composed mainly of infiltrative tumor $(64 \%)$. Although approximately half $(51 \%)$ of the samples with no fluorescence did not show distinct tumor cells, the remaining approximate half $(49 \%)$ of the samples were composed almost exclusively of infiltrative tumor. Accordingly, Stummer et al..$^{32,35}$ found in their 2 studies, which analyzed newly diagnosed malignant gliomas, that samples with strong fluorescence consisted mainly of compact tumor, whereas samples with vague fluorescence corresponded predominantly to infiltrative tumor. This observation was confirmed in a recent study conducted by Coburger et al. ${ }^{6}$ that also included recurrent GBMs. Similarly, the group of Díez Valle et al.,12 found compact tumor in all analyzed tissue samples from newly diagnosed and recurrent GBMs with strong fluorescence, and in approximately $90 \%$ of their samples with vague fluorescence, they found infiltrative tumor.

\section{Histopathological Criteria of Malignancy}

Cell Density. First, we found in our study that samples with strong fluorescence frequently had high cell density $(62 \%)$, samples with vague fluorescence usually had low-to-moderate cell density (77\%), and samples with no 
fluorescence were characterized by normal-to-moderate cell density (97\%). Accordingly, in 2000, Stummer et al. ${ }^{32}$ first observed by analyzing smear preparations of samples from 52 patients with a newly diagnosed GBM that strongly fluorescing tissue mostly contained tissue with high cell density, whereas vague fluorescence corresponded frequently to tissue with low cell density. In 2014, these authors confirmed this finding in another study by using a conventional diagnostic histopathological workup of their collected tissue specimens. ${ }^{35}$ Similarly, in a study of newly diagnosed and of recurrent WHO Grade III and IV gliomas using 4 fluorescence levels (Grade 0-3), Lau et al. ${ }^{15}$ reported recently that the highest 5-ALA fluorescence intensity (Grade 3 ) correlated significantly with the highest degree of tumor cellularity (Grade 3).

Nuclear Pleomorphism. Furthermore, the majority of samples with strong, vague, or no fluorescence were characterized by high (77\%), low-to-high (94\%), and normalto-moderate $(90 \%)$ nuclear pleomorphism, respectively. To our knowledge, before our study, nuclear pleomorphism had not been analyzed separately in these 3 5-ALA-induced fluorescence levels of newly diagnosed GBMs.

Mitotic Activity. Moreover, most samples with strong fluorescence were characterized by some-to-many mitotic figures $(86 \%)$, specimens with vague fluorescence by few mitotic figures (57\%), and samples with no fluorescence by no-to-few mitotic figures (76\%). Again, to our knowledge, mitotic activity had not been investigated as a separate parameter in these 3 fluorescence levels of newly diagnosed GBMs before our study.

Microvascular Proliferation and Necrosis. Both diagnostic histopathological GBM criteria, microvascular proliferation (95\%) and necrosis (76\%), were usually detected in samples with strong fluorescence, and at least 1 diagnostic histopathological GBM criterion consisting of microvascular proliferation $(72 \%)$ was found in the majority of samples with vague fluorescence. In contrast, diagnostic histopathological GBM criteria were frequently absent in samples with no fluorescence (absence of microvascular proliferation and focal necrosis were found in $76 \%$ and $93 \%$ of the samples, respectively). To our knowledge, ours is the first study to have analyzed separately the presence of microvascular proliferation and focal necrosis in samples of newly diagnosed GBMs with strong, vague, or no fluorescence. It is notable that in our study, necrosis occurred only focally within the tissue samples and was never the only histopathological feature. In general, samples that contain only necrotic tissue do not exhibit significant 5 -ALA-induced fluorescence. ${ }^{8,40}$ In a study that also included recurrent GBMs, however, the group of Díez Valle et al. ${ }^{7,12}$ reported similar findings and observed histopathological GBM criteria in all samples with strong fluorescence, in $8.2 \%$ of samples with vague fluorescence, and in none of the samples with no fluorescence. In another study that included 11 patients with a newly diagnosed GBM and in which 4 fluorescence levels were used, Roberts et al. ${ }^{27}$ observed a significant correlation of the fluorescence levels with a histopathological score that is based on WHO grading and included also necrosis and endothelial proliferation as parameters (histopathological score IV).

\section{Proliferation Rate}

We also found for the first time that the proliferation rate significantly correlated with the 3 fluorescence levels in newly diagnosed GBMs. In this context, the mean MIB$1 \mathrm{LI}$ was high in samples with strong fluorescence $(28.3 \%)$, moderate in samples with vague fluorescence (16.7\%), and low in nonfluorescing samples $(8.8 \%)$. In the 2 studies performed by Díez Valle et al.,,12 which also included recurrent GBMs, the authors observed significant differences in the Ki-67 LI in the 3 fluorescence levels. ${ }^{7,12}$ However, we found in our study markedly higher values for the proliferation rate in samples with 1 of the 3 fluorescence levels than in those in these other 2 studies. ${ }^{7,12}$ One possible explanation for this finding is that the proliferation rate was assessed in our study in areas of "hot spots," whereas in the 2 other studies, an automatic measurement technique was applied. ${ }^{7,12}$ Interestingly, another recent study in which 4 fluorescence levels were used did not find significant differences in the proliferation rate between tissue samples of strong, moderate, weak, or no fluorescence. ${ }^{9}$ Nevertheless, these data have to be considered with caution because of the small number $(n=13)$ of included patients who were suffering from a newly diagnosed GBM. ${ }^{9}$

\section{Microvessel Density}

We detected a significantly higher microvessel density in samples with strong fluorescence (125.5 vessels/0.25 $\left.\mathrm{mm}^{2}\right)$ than in those with vague $\left(82.8\right.$ vessels $\left./ 0.25 \mathrm{~mm}^{2}\right)$ or no $\left(68.6\right.$ vessels $\left./ 0.25 \mathrm{~mm}^{2}\right)$ fluorescence. Nevertheless, no significant difference in terms of microvessel density was found in samples with vague fluorescence and those with no fluorescence. To our knowledge, ours is the first study in a large series of patients with a newly diagnosed GBM to report a significantly higher microvessel density in areas of strong fluorescence than that in those with vague or no fluorescence.

\section{Surgical Benefits and Limits of 5-ALA in Newly Diagnosed GBMs}

According to our data, visible 5-ALA-induced fluorescence serves as a clinically reliable and immediately available marker for the presence of tumor tissue during surgery in patients with a newly diagnosed GBM. In addition, the different fluorescence levels are unaffected by brain shift and enable intraoperative estimation of the degree of histopathological "tissue aggressiveness" in different intratumoral areas. As a consequence, knowledge of the malignancy of this tissue can guide the neurosurgeon to achieve the surgical goal of maximal safe resection of GBMs, which depends on tumor localization and is a requirement for inclusion in specific clinical trials, such as most ongoing immunotherapy studies. ${ }^{29}$ In GBMs with eloquent tumor localization, strong fluorescence enables the neurosurgeon to identify and resect the most histopathologically aggressive intratumoral areas when it is safe to do so. The presence of vague fluorescence in such tumors might serve as a warning sign for the neurosurgeon that presumably infiltrative tumor tissue has already been reached during the resection. ${ }^{35}$ In GBMs with noneloquent tumor localization, vague fluorescence is especially useful for visualizing the presence of tissue that consists mostly 
of infiltrative tumor and still usually shows a significantly increased proliferation rate and microvessel density. Aldave et al. ${ }^{2}$ found prolonged overall survival in patients after gross-total resection of a newly diagnosed GBM and additional complete removal of all 5-ALA-induced tissue fluorescence compared to patients with residual fluorescing tissue. Therefore, we recommend that in patients with a GBM with noneloquent tumor localization, neurosurgeons should resect this residual fluorescing tissue, which usually consists of vague fluorescence, in addition to the tissue with strong fluorescence, if it can be done safely, to positively influence patient prognosis. It is notable, however, that techniques such as intraoperative mapping/ stimulation and/or navigation with DTI/fMRI data are indispensable for ensuring maximal safety of these 5-ALA fluorescence-guided procedures.

It is unfortunate, however, that the 5-ALA technique cannot reliably indicate the absence of tumor tissue in newly diagnosed GBMs by the lack of fluorescence, and this issue represents a major limitation of the method. According to our data, neurosurgeons will leave behind predominantly infiltrative tumor tissue with a relatively low proliferation rate and microvessel density in approximately half of the cases despite complete resection of all fluorescing tissue. Despite this limitation of 5-ALA, this method enables the neurosurgeon to identify additional infiltrative tumor tissue beyond the contrast-enhancing area on MRI, which is currently considered to be the accepted surgical target for resection of GBMs. ${ }^{8,35}$ To improve the visualization of tumor tissue with a lack of fluorescence, the complementary spectroscopic measurement of 5-ALAinduced PpIX accumulation during surgery seems to be an especially promising approach according to data from the first studies. ${ }^{26,35,38}$ As a consequence, the value of this approach has to be investigated further to clarify if it is clinically reliable for also identifying nonfluorescing infiltrative tumor tissue in newly diagnosed GBMs.

\section{Limitations of This Study}

Specific limitations of this study have to be mentioned. First, the 3 fluorescence levels applied in our study were assessed semiquantitatively by the performing neurosurgeon during surgery; thus, this classification is subjective. In addition, it must be assumed that there exists a smooth transition across the fluorescence spectrum without distinct break points; therefore, the actual transition between each fluorescence level is arbitrary. These are major limitations of all studies that use a semiquantitative classification system to assess fluorescence. It is interesting that despite these limitations, we found almost perfect interobserver agreement between a group of neurosurgeons who separately classified various fluorescence levels on the same intraoperative photographs. One possible explanation for this very high interobserver agreement is that for the past 9 years, our department has had a high caseload of 5-ALA fluorescence-guided surgeries; thus, our neurosurgeons have a lot of experience with these procedures. However, to confirm the very high interobserver agreement on classification of the 3 fluorescence levels that we observed in our study, multicentric confirmatory studies are required. Furthermore, our study lacks data concerning the quanti- fication of fluorescence because we performed only semiquantitative assessment of fluorescence. As a consequence, quantitative measurement of 5-ALA-induced PpIX accumulation in a large series of newly diagnosed GBMs, especially by intraoperative spectroscopic analysis ${ }^{26,35,38}$ and systematic histopathological correlation, should be performed in further studies. Our study also lacks data concerning the potential influence of treatment-associated tissue changes on 5-ALA-induced fluorescence because recurrent GBMs were not included. However, this study was designed primarily to investigate the precise histopathological correlate of the different fluorescence levels in newly diagnosed GBMs. Therefore, a comprehensive histopathological analysis of the different fluorescence levels in recurrent GBMs should be performed in future studies. Last, a systematic topographical correlation study of the different fluorescence levels with neuronavigationbased imaging data was not systematically conducted. Nevertheless, such an analysis was not within the scope of this study. Optimally, the different fluorescence levels should be topographically correlated with specific intratumoral areas (e.g., contrast-enhancing tumor, nonenhancing FLAIR/T2-weighted hyperintense lesion) and verified by intraoperative MRI to prevent the consequences of potential brain shift.

\section{Conclusions}

In this prospective study, we systematically analyzed the precise histopathological correlate of 5-ALA-induced fluorescence in a large series of newly diagnosed GBMs. We found a very high PPV of both strong and vague fluorescence for the presence of tumor tissue $(100 \%$ and $94 \%$, respectively). However, tumor tissue was still present in approximately half of the cases despite the lack of fluorescence, which resulted in a low NPV (51\%). While strong fluorescence supports the neurosurgeon in identifying compact tumor independent of brain shift, vague fluorescence is useful for visualizing mainly infiltrative tumor during 5-ALA fluorescence-guided surgery to improve the extent of resection. Furthermore, histopathological criteria of malignancy and proliferation rate significantly correlated with the 3 fluorescence levels, and microvessel density was significantly higher in areas with strong fluorescence than in those with vague or no fluorescence. Additional studies should clarify if the promising approach of complementary spectroscopic measurement of 5-ALAinduced PpIX accumulation would result in improved visualization of nonfluorescing infiltrative tumor tissue to increase the low NPV of visible fluorescence.

\section{Acknowledgments}

We thank Dr. Harald Heinzl, Core Unit for Medical Statistics and Informatics, for statistical advice and Irene Leisser, Elisabeth Mitterer, Anita Nguyen, Gerda Ricken, and Brigitte HammerSchmiedel for technical assistance, including preparation of tissue specimens. This study was performed within the $\mathrm{PhD}$ thesis project of Clinical Neuroscience (CLINS) (B.K.) at the Medical University Vienna.

\section{References}

1. Albert FK, Forsting M, Sartor K, Adams HP, Kunze S: Early 
postoperative magnetic resonance imaging after resection of malignant glioma: objective evaluation of residual tumor and its influence on regrowth and prognosis. Neurosurgery 34:45-61, 1994

2. Aldave G, Tejada S, Pay E, Marigil M, Bejarano B, Idoate MA, et al: Prognostic value of residual fluorescent tissue in glioblastoma patients after gross total resection in 5-aminolevulinic acid-guided surgery. Neurosurgery 72:915-921, 2013

3. Assimakopoulou M, Sotiropoulou-Bonikou G, Maraziotis T, Papadakis N, Varakis I: Microvessel density in brain tumors. Anticancer Res 17:4747-4753, 1997

4. Belloch JP, Rovira V, Llácer JL, Riesgo PA, Cremades A: Fluorescence-guided surgery in high grade gliomas using an exoscope system. Acta Neurochir (Wien) 156:653-660, 2014

5. Bush NAO, Chang SM, Berger MS: Current and future strategies for treatment of glioma. Neurosurg Rev 40:1-14, 2017

6. Coburger J, Engelke J, Scheuerle A, Thal DR, Hlavac M, Wirtz CR, et al: Tumor detection with 5-aminolevulinic acid fluorescence and Gd-DTPA-enhanced intraoperative MRI at the border of contrast-enhancing lesions: a prospective study based on histopathological assessment. Neurosurg Focus 36(2):E3, 2014

7. Díez Valle R, Tejada Solis S, Idoate Gastearena MA, García de Eulate R, Domínguez Echávarri P, Aristu Mendiroz J: Surgery guided by 5 -aminolevulinic fluorescence in glioblastoma: volumetric analysis of extent of resection in singlecenter experience. J Neurooncol 102:105-113, 2011

8. Hadjipanayis CG, Widhalm G, Stummer W: What is the surgical benefit of utilizing 5-aminolevulinic acid for fluorescence-guided surgery of malignant gliomas? Neurosurgery 77:663-673, 2015

9. Hauser SB, Kockro RA, Actor B, Sarnthein J, Bernays RL: Combining 5-aminoevulinic fluorescence and intraoperative magnetic resonance imaging in glioblastoma surgery: a histology-based evaluation. Neurosurgery 78:475-483, 2015

10. Hefti M, von Campe G, Moschopulos M, Siegner A, Looser H, Landolt H: 5-aminolaevulinic acid-induced protoporphyrin IX fluorescence in high-grade glioma surgery. A one-year experience at a single institution. Swiss Med Wkly 138:180185,2008

11. Hsu CY, Ho DM, Yang CF, Chiang H: Interobserver reproducibility of MIB-1 labeling index in astrocytic tumors using different counting methods. Mod Pathol 16:951-957, 2003

12. Idoate MA, Díez Valle R, Echeveste J, Tejada S: Pathological characterization of the glioblastoma border as shown during surgery using 5-aminolevulinic acid-induced fluorescence. Neuropathology 31:575-582, 2011

13. Kamp MA, Krause Molle Z, Munoz-Bendix C, Rapp M, Sabel M, Steiger HJ, et al: Various shades of red-a systematic analysis of qualitative estimation of ALA-derived fluorescence in neurosurgery. Neurosurg Rev [epub ahead of print], 2016

14. Kubben PL, ter Meulen KJ, Schijns OEMG, ter Laak-Poort MP, van Overbeeke JJ, van Santbrink H: Intraoperative MRIguided resection of glioblastoma multiforme: a systematic review. Lancet Oncol 12:1062-1070, 2011

15. Lau D, Hervey-Jumper SL, Chang S, Molinaro AM, McDermott MW, Phillips JJ, et al: A prospective Phase II clinical trial of 5-aminolevulinic acid to assess the correlation of intraoperative fluorescence intensity and degree of histologic cellularity during resection of high-grade gliomas. J Neurosurg 124:1300-1309, 2016

16. Li YM, Suki D, Hess K, Sawaya R: The influence of maximum safe resection of glioblastoma on survival in 1229 patients: Can we do better than gross-total resection? J Neurosurg 124:977-988, 2016

17. Louis DN, Ohgaki H, Wiestler OD, Cavenee WK (eds):
WHO Classification of Tumours of the Central Nervous System, Revised, ed 4. Lyon, France: International Agency for Research on Cancer, 2016

18. Mert A, Gan LS, Knosp E, Sutherland GR, Wolfsberger S: Advanced cranial navigation. Neurosurgery 72 (Suppl 1):43-53, 2013

19. Orringer D, Lau D, Khatri S, Zamora-Berridi GJ, Zhang K, Wu C, et al: Extent of resection in patients with glioblastoma: limiting factors, perception of resectability, and effect on survival. J Neurosurg 117:851-859, 2012

20. Panciani PP, Fontanella M, Garbossa D, Agnoletti A, Ducati A, Lanotte M: 5-aminolevulinic acid and neuronavigation in high-grade glioma surgery: results of a combined approach. Neurocirugia (Astur) 23:23-28, 2012

21. Panciani PP, Fontanella M, Schatlo B, Garbossa D, Agnoletti A, Ducati A, et al: Fluorescence and image guided resection in high grade glioma. Clin Neurol Neurosurg 114:37-41, 2012

22. Preusser M, Charles Janzer R, Felsberg J, Reifenberger G, Hamou MF, Diserens AC, et al: Anti-O6-methylguaninemethyltransferase (MGMT) immunohistochemistry in glioblastoma multiforme: observer variability and lack of association with patient survival impede its use as clinical biomarker. Brain Pathol 18:520-532, 2008

23. Preusser M, de Ribaupierre S, Wöhrer A, Erridge SC, Hegi M, Weller M, et al: Current concepts and management of glioblastoma. Ann Neurol 70:9-21, 2011

24. Preusser M, Heinzl H, Gelpi E, Schonegger K, Haberler C, Birner P, et al: Histopathologic assessment of hot-spot microvessel density and vascular patterns in glioblastoma: poor observer agreement limits clinical utility as prognostic factors: a translational research project of the European Organization for Research and Treatment of Cancer Brain Tumor Group. Cancer 107:162-170, 2006

25. Raghavan R, Steart PV, Weller RO: Cell proliferation patterns in the diagnosis of astrocytomas, anaplastic astrocytomas and glioblastoma multiforme: a Ki-67 study. Neuropathol Appl Neurobiol 16:123-133, 1990

26. Richter J, Haj-Hosseini N, Hallbeck M, Wårdell K: Combination of hand-held probe and microscopy for fluorescence guided surgery in the brain tumor marginal zone. Photodiagnosis Photodyn Ther [epub ahead of print], 2017

27. Roberts DW, Valdés PA, Harris BT, Fontaine KM, Hartov A, Fan X, et al: Coregistered fluorescence-enhanced tumor resection of malignant glioma: relationships between $\delta$ aminolevulinic acid-induced protoporphyrin IX fluorescence, magnetic resonance imaging enhancement, and neuropathological parameters. Clinical article. J Neurosurg 114:595603,2011

28. Sanai N, Polley MY, McDermott MW, Parsa AT, Berger MS: An extent of resection threshold for newly diagnosed glioblastomas. J Neurosurg 115:3-8, 2011

29. Schuster J, Lai RK, Recht LD, Reardon DA, Paleologos NA, Groves MD, et al: A phase II, multicenter trial of rindopepimut (CDX-110) in newly diagnosed glioblastoma: the ACT III study. Neuro Oncol 17:854-861, 2015

30. Senft C, Bink A, Franz K, Vatter H, Gasser T, Seifert V: Intraoperative MRI guidance and extent of resection in glioma surgery: a randomised, controlled trial. Lancet Oncol 12:997-1003, 2011

31. Serra C, Stauffer A, Actor B, Burkhardt JK, Ulrich NHB, Bernays RL, et al: Intraoperative high frequency ultrasound in intracerebral high-grade tumors. Ultraschall Med 33:E306-E312, 2012

32. Stummer W, Novotny A, Stepp H, Goetz C, Bise K, Reulen HJ: Fluorescence-guided resection of glioblastoma multiforme by using 5-aminolevulinic acid-induced porphyrins: a prospective study in 52 consecutive patients. J Neurosurg 93:1003-1013, 2000 
33. Stummer W, Pichlmeier U, Meinel T, Wiestler OD, Zanella F, Reulen HJ: Fluorescence-guided surgery with 5-aminolevulinic acid for resection of malignant glioma: a randomised controlled multicentre phase III trial. Lancet Oncol 7:392401, 2006

34. Stummer W, Stepp H, Möller G, Ehrhardt A, Leonhard M, Reulen HJ: Technical principles for protoporphyrin-IX-fluorescence guided microsurgical resection of malignant glioma tissue. Acta Neurochir (Wien) 140:995-1000, 1998

35. Stummer W, Tonn JC, Goetz C, Ullrich W, Stepp H, Bink A, et al: 5-Aminolevulinic acid-derived tumor fluorescence: the diagnostic accuracy of visible fluorescence qualities as corroborated by spectrometry and histology and postoperative imaging. Neurosurgery 74:310-320, 2014

36. Stupp R, Mason WP, van den Bent MJ, Weller M, Fisher B, Taphoorn MJB, et al: Radiotherapy plus concomitant and adjuvant temozolomide for glioblastoma. $\mathbf{N}$ Engl J Med 352:987-996, 2005

37. Tonn JC, Stummer W: Fluorescence-guided resection of malignant gliomas using 5-aminolevulinic acid: practical use, risks, and pitfalls. Clin Neurosurg 55:20-26, 2008

38. Valdés PA, Leblond F, Kim A, Harris BT, Wilson BC, Fan $\mathrm{X}$, et al: Quantitative fluorescence in intracranial tumor: implications for ALA-induced PpIX as an intraoperative biomarker. J Neurosurg 115:11-17, 2011

39. Vogelbaum MA, Jost S, Aghi MK, Heimberger AB, Sampson JH, Wen PY, et al: Application of novel response/progression measures for surgically delivered therapies for gliomas: Response Assessment in Neuro-Oncology (RANO) Working Group. Neurosurgery 70:234-244, 2012

40. Widhalm G: Intra-operative visualization of brain tumors with 5-aminolevulinic acid-induced fluorescence. Clin Neuropathol 33:260-278, 2014

41. Widhalm G, Kiesel B, Woehrer A, Traub-Weidinger T, Preusser M, Marosi C, et al: 5-Aminolevulinic acid induced fluorescence is a powerful intraoperative marker for precise histopathological grading of gliomas with non-significant contrast-enhancement. PLoS One 8: 76988,2013

42. Widhalm G, Krssak M, Minchev G, Wöhrer A, Traub-
Weidinger T, Czech T, et al: Value of ${ }^{1} \mathrm{H}$-magnetic resonance spectroscopy chemical shift imaging for detection of anaplastic foci in diffusely infiltrating gliomas with non-significant contrast-enhancement. J Neurol Neurosurg Psychiatry 82:512-520, 2011

43. Widhalm G, Wolfsberger S, Minchev G, Wöhrer A, Krssak M, Czech T, et al: 5-Aminolevulinic acid is a promising marker for detection of anaplastic foci in diffusely infiltrating gliomas with nonsignificant contrast enhancement. Cancer 116:1545-1552, 2010

44. Yamahara T, Numa Y, Oishi T, Kawaguchi T, Seno T, Asai A, et al: Morphological and flow cytometric analysis of cell infiltration in glioblastoma: a comparison of autopsy brain and neuroimaging. Brain Tumor Pathol 27:81-87, 2010

\section{Disclosures}

Stefan Wolfsberger is currently an educational consultant and a technological advisory board member of Medtronic.

\section{Author Contributions}

Conception and design: Widhalm, Kiesel. Acquisition of data: Widhalm, Kiesel, Mischkulnig, Woehrer, Martinez-Moreno, Millesi, Mallouhi, Czech, Preusser, Wolfsberger, Knosp. Analysis and interpretation of data: Widhalm, Kiesel, Woehrer, Martinez-Moreno, Millesi, Mallouhi, Czech, Preusser, Hainfellner, Wolfsberger, Knosp. Drafting the article: Widhalm, Kiesel. Critically revising the article: all authors. Reviewed submitted version of manuscript: all authors. Approved the final version of the manuscript on behalf of all authors: Widhalm. Statistical analysis: Widhalm, Kiesel, Mischkulnig, Millesi. Administrative/technical/material support: Widhalm, Kiesel, Mischkulnig, Woehrer, MartinezMoreno, Mallouhi, Czech, Preusser, Hainfellner, Knosp. Study supervision: Widhalm, Hainfellner.

\section{Correspondence}

Georg Widhalm, Department of Neurosurgery, Medical University Vienna, Waehringer Guertel 18-20, A-Vienna 1097, Austria. email: georg.widhalm@meduniwien.ac.at. 